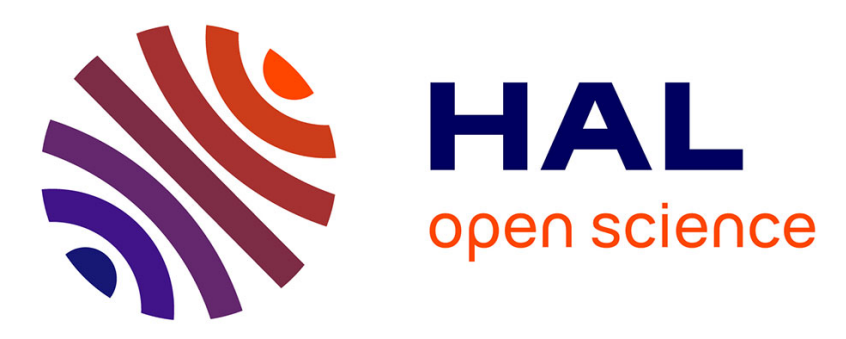

\title{
Reluctant Cross-Metathesis Reactions: The Highly Beneficial Effect of Microwave Irradiation
}

Antoine Michaut, Thomas Boddaert, Yoann Coquerel, Jean Rodriguez

\section{To cite this version:}

Antoine Michaut, Thomas Boddaert, Yoann Coquerel, Jean Rodriguez. Reluctant Cross-Metathesis Reactions: The Highly Beneficial Effect of Microwave Irradiation. Synthesis: Journal of Synthetic Organic Chemistry, 2007, 18, pp.2867-2871. 10.1055/s-2007-983825 . hal-00676779

\section{HAL Id: hal-00676779 \\ https://hal.science/hal-00676779}

Submitted on 6 Mar 2012

HAL is a multi-disciplinary open access archive for the deposit and dissemination of scientific research documents, whether they are published or not. The documents may come from teaching and research institutions in France or abroad, or from public or private research centers.
L'archive ouverte pluridisciplinaire HAL, est destinée au dépôt et à la diffusion de documents scientifiques de niveau recherche, publiés ou non, émanant des établissements d'enseignement et de recherche français ou étrangers, des laboratoires publics ou privés. 


\title{
Reluctant Cross-Metathesis Reactions: a Highly Beneficial Effect of Micro- wave Irradiation
}

\author{
Antoine Michaut, Thomas Boddaert, Yoann Coquerel,* Jean Rodriguez* \\ Université Paul Cézanne (Aix-Marseille III), CNRS, UMR 6178, Campus universitaire de St Jérôme, Service 531, 13397 Marseille \\ Cedex 20, France. \\ Fax: +33(0)491289187 \\ E-mail: yoann.coquerel@univ-cezanne.fr and jean.rodriguez@univ-cezanne.fr
}

\begin{abstract}
The beneficial effect of microwave irradiation $v s$ classical thermal conditions is demonstrated through a series of comparative cross-metathesis reactions.
\end{abstract}

Key words: Carbene complexes, Catalysis, Cross-coupling, Metathesis, Olefination.

The establishment of olefin metathesis as a powerfull synthetic tool for $\mathrm{C}=\mathrm{C}$ bond formation is essentially due to the initial discovery of the Schrock molybdenumbased catalyst, followed by the extensive development of the ruthenium-based catalyst such as $\mathbf{1 a , b}$ and their derivatives introduced by Grubbs and co-workers. More specifically, cross-metathesis (CM) of simple olefins has now become one of the method of choice to access substituted olefins, although its development has been somewhat delayed when compared to Ring Closing Metathesis (RCM) and Ring Opening Metathesis Polymerization (ROMP) due to initial functional group compatibility problems. ${ }^{1}$ In some cases, and in particular with electro-deficient olefins, the CM reaction is less effective and requires specific conditions to prevent catalyst deactivation. Among them, addition of $\mathrm{Cy}_{2} \mathrm{BCl}$ or $\mathrm{Ti}(\mathrm{O} i \mathrm{Pr})_{4}$ have proven useful with substrates bearing a Lewis base functional group. ${ }^{2,3 h}$ The CM reactions with reluctant partners such as acrylonitrile derivatives have been the topic of several studies, and specific conditions as well as more reactive phosphine-free catalyst have been developped. ${ }^{3}$ Recently, some reports appeared on the dramatic improvement in reaction rates and yields in microwave-assisted olefin metathesis reactions. ${ }^{4}$

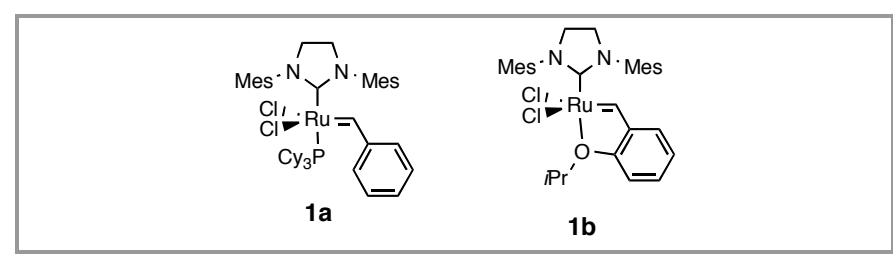

Figure 1 Metathesis catalysts used.

In connection with our studies on 1,3-dicarbonyl derivatives, we recently prepared a number of allylic derivatives by $\mathrm{CM}$ reactions with various functionalized olefins using catalysts $\mathbf{1 a}, \mathbf{b}$. If the desired products could indeed be obtained under classical thermal conditions, reaction times were rather long, and in some cases the yields were modest to very low, probably due to the polar functionnalities present on the substrate. With in mind the precedents of microwave $(\mu \mathrm{W})$ irradiation during metathesis reactions, ${ }^{4}$ we tested this method of activation in the cases of our reluctant subtrates. We observed a highly beneficial effect on the rate of the $\mathrm{CM}$ reactions under $\mu \mathrm{W}$ irradiation, and our results are reported herein (Table 1). It should be noted that $\mu \mathrm{W}$ assisted CM reactions have been scarcely reported, and only with ethyl acrylate, ${ }^{4 \mathrm{q}}$ for the homodimerization of $\mathrm{N}$-allyl amino acids, ${ }^{4 \mathrm{p}}$ and very recently with peptides. ${ }^{4 \mathrm{r}}$ We initiated our study with the cross-metathesis of allylic derivatives and allyl(trimethyl)silane (allylTMS, 3a). The $\beta$-ketoamide 2a is reluctant to undergo $\mathrm{CM}$ with allylTMS (3a) under classical thermal conditions, resulting in poor conversion leading to a low yield of the desired cross-coupled product, always accompanied by substantial amounts of products resulting from the homocoupling of the substrate and partial isomerisation of the allylTMS (3a) to the vinyl silane (Table 1, entry 1). The same reaction performed under $\mu \mathrm{W}$ irradiation resulted in a dramatic increase in the rate of the $\mathrm{CM}$ to give $60 \%$ of the expected cross-coupling product $(87 \%$ conversion) after only 30 seconds at $60^{\circ} \mathrm{C}$ (entry 1 ). The isomerisation of allylTMS (3a) could be completely suppressed by addition of $10 \%$ of 1,4-benzoquinone. ${ }^{5}$ Going down to $0.6 \mathrm{~mol} \%$ of catalyst is still productive yielding $38 \%$ of cross-product after 30 minutes of irradiation (entry 2). A similar accelerating effect was observed for the cross-coupling reactions of the $\beta$ ketosulfone 2b with allylTMS (3a) which resulted in $68 \%$ yield of the expected olefin after 35 minutes of irradiation compared to $51 \%$ after 24 hours under thermal conditions (entry 3). The beneficial effect of $\mu \mathrm{W}$ irradiation is even more striking with the diketone $\mathbf{2 c}$, as the $\mathrm{CM}$ product is obtained in $75 \%$ yield after $10 \mathrm{~min}$ utes, while $10 \%$ yield is obtained after 16 hours at 100 ${ }^{\circ} \mathrm{C}$ without irradiation (entry 4). The $\mathrm{CM}$ of the more sensitive and probably more chelating nitroketone 2d required the addition of $\mathrm{Ti}(\mathrm{O} i \mathrm{Pr})_{4}$ and $\mu \mathrm{W}$ irradiation to proceed smoothly (entry 5). The cross-coupling of the homoallylic bromide 2e and allylTMS (3a) under thermal conditions gave a very sluggish reaction while $\mu \mathrm{W}$ irradiation allowed the isolation of the alkene $4 \mathrm{e}$ in $77 \%$ yield after only 40 seconds (entry 6 ). Interestingly, scaling up the reaction to $15 \mathrm{mmol}$ allowed to cut down the amount of catalyst to $0.15 \%$ to produce $69 \%$ of $4 \mathrm{e}$ after 3 minutes of irradiation (entry 7). This correspond to an exceptionnally high TON of 460 for catalyst 1a in CM. ${ }^{6}$ The nitroalkene $\mathbf{2 f}$ also underwent CM with allylTMS (3a) in decent yield after 30 seconds of irradiation (entry 8). Hexene (3b) and allyl acetate (3c) were also used successfully as $\mathrm{CM}$ partners under $\mu \mathrm{W}$ irradiation with the $\beta$-ketoester $2 \mathrm{~g}$ to give good $Z$ selectivity in the latter 
case (entries 9 and 10). When acrylonitrile (3d) is used as the CM partner with the $\beta$-ketoester $\mathbf{2 h}$, catalyst $\mathbf{1 b}$ was by far superior to $\mathbf{1 a},{ }^{3}$ as illustrated in entries 11-15. For both thermal and $\mu \mathrm{W}$ conditions, the reactions were performed at $100{ }^{\circ} \mathrm{C}$ with the same catalyst loading and times. Comparable results were obtained in dichloroethane or dichloromethane (compare entries 11 with 12 , and 13 with 14). However, with catalyst 1a (entries 11 and 12) slightly lower yields were obtained under $\mu \mathrm{W}$ irradiation, probably due to faster decomposition of the catalyst. The decomposition of catalyst $\mathbf{1 b}$ under the reaction conditions is also evidenced in entries 14 and 15, as for the same total amount of catalyst, better results are obtained by introduction of the catalyst in two portions. As previously reported, ${ }^{3} \mathrm{CM}$ reactions with acrylonitrile (3d) exhibit a pronounced $Z$ selectivity of the product.

\begin{tabular}{|c|c|c|c|c|c|c|c|c|}
\hline \multirow[b]{2}{*}{ Entry } & \multirow[b]{2}{*}{ Substrate } & \multirow[b]{2}{*}{ Olefin } & \multirow[b]{2}{*}{$\begin{array}{l}\text { Catalyst } \\
(\%)\end{array}$} & \multirow[b]{2}{*}{ Product } & \multicolumn{2}{|c|}{ Thermal conditions } & \multicolumn{2}{|c|}{ Microwave irradiation } \\
\hline & & & & & Conditions & $\begin{array}{l}\text { Yield } \\
(\%) ; \\
E / Z \text { ratio }^{\text {a }} \\
\end{array}$ & Conditions $^{\mathrm{b}}$ & $\begin{array}{l}\text { Yield } \\
(\%) ; \\
\text { E/Z ratio } \\
\end{array}$ \\
\hline 1 & $2 \mathbf{a}$ & $ح_{\mathbf{3 a}}^{\mathrm{SiMe}_{3}}$ & $1 \mathbf{a}(2)$ & & Neat, $90^{\circ} \mathrm{C}, 16 \mathrm{~h}$ & $14 ; 3: 1$ & $\begin{array}{l}\text { Neat, } 60{ }^{\circ} \mathrm{C}, 30 \mathrm{~s} \text {, } \\
10 \% 1,4- \\
\text { benzoquinone }\end{array}$ & $60,3: 1$ \\
\hline 2 & $2 a$ & 3a & 1a $(0.6)$ & $4 a$ & Not tested & - & $\begin{array}{l}\text { Neat, } 60{ }^{\circ} \mathrm{C}, 30 \mathrm{~s} \text {, } \\
10 \% 1,4- \\
\text { benzoquinone }\end{array}$ & $38,1: 1$ \\
\hline 3 & & $3 a$ & $1 a(3)$ & & Neat, $90{ }^{\circ} \mathrm{C}, 24 \mathrm{~h}$ & $51 ; 1: 1$ & $\begin{array}{l}\text { Neat, } 60{ }^{\circ} \mathrm{C}, 35 \\
\text { min }\end{array}$ & $68,1: 1$ \\
\hline 4 & & $3 a$ & $1 \mathbf{a}(2)$ & & $\begin{array}{l}\text { Neat, } 100{ }^{\circ} \mathrm{C}, 16 \\
\mathrm{~h}\end{array}$ & $10 ; 1.8: 1$ & $\begin{array}{l}\text { Neat, } 90{ }^{\circ} \mathrm{C}, 10 \\
\text { min }\end{array}$ & $75 ; 1.8: 1$ \\
\hline 5 & & $3 a$ & $1 \mathbf{a}(4)$ & & $\begin{array}{l}\mathrm{CH}_{2} \mathrm{Cl}_{2}, 40{ }^{\circ} \mathrm{C}, \\
15 \% \mathrm{Ti}(\mathrm{O} i \mathrm{Pr})_{4}, \\
16 \mathrm{~h}\end{array}$ & $40 ; 1.8: 1$ & $\begin{array}{l}\mathrm{CH}_{2} \mathrm{Cl}_{2}, 60{ }^{\circ} \mathrm{C}, \\
15 \% \mathrm{Ti}(\mathrm{O} i \mathrm{Pr})_{4}, \\
40 \mathrm{~min}\end{array}$ & $62 ; 1.8: 1$ \\
\hline 6 & & 3a & $\mathbf{1 a}(0.8)$ & & Neat, $90{ }^{\circ} \mathrm{C}, 8 \mathrm{~h}$ & $6 ; \mathrm{nd}^{\mathrm{c}}$ & Neat, $60{ }^{\circ} \mathrm{C}, 40 \mathrm{~s}$ & $77 ; 1.9: 1$ \\
\hline 7 & $2 e$ & $3 a$ & $1 \mathbf{a}(0.15)$ & $4 e$ & Not tested & - & $\begin{array}{l}\text { Neat, } 60{ }^{\circ} \mathrm{C}, 3 \\
\text { min }\end{array}$ & $69 ; 1.9: 1$ \\
\hline 8 & & $3 a$ & $\mathbf{1 a}(1.5)$ & $\mathrm{Me}_{3} \mathrm{Si}^{\prime}$ & Not tested & - & Neat, $60{ }^{\circ} \mathrm{C}, 30 \mathrm{~s}$ & $57 ; 2.3: 1$ \\
\hline 9 & & $\begin{array}{c}\gamma / r_{3} \\
3 \mathbf{b}\end{array}$ & 1a (2) & & Neat, $90{ }^{\circ} \mathrm{C}, 16 \mathrm{~h}$ & $50 ; 4: 1$ & Neat, $60{ }^{\circ} \mathrm{C}, 75 \mathrm{~s}$ & $68 ; 4: 1$ \\
\hline 10 & $2 \mathrm{~g}$ & $\widehat{3 c}^{O A C}$ & 1a (3) & & $\begin{array}{l}\mathrm{CH}_{2} \mathrm{Cl}_{2}, 40{ }^{\circ} \mathrm{C}, \\
12 \mathrm{~h}\end{array}$ & $41 ; 1: 5.7$ & $\begin{array}{l}\mathrm{CH}_{2} \mathrm{Cl}_{2}, 60{ }^{\circ} \mathrm{C}, \\
15 \mathrm{~min}\end{array}$ & $78 ; 1: 5.7$ \\
\hline 11 & & $\widehat{C}_{\mathrm{CN}}$ & $1 \mathbf{a}(3+1)$ & & $\begin{array}{l}\mathrm{CH}_{2} \mathrm{Cl}_{2}, 100{ }^{\circ} \mathrm{C}, \\
20+10 \mathrm{~min}\end{array}$ & $35 ; 1: 3.8$ & $\begin{array}{l}\mathrm{CH}_{2} \mathrm{Cl}_{2}, 100{ }^{\circ} \mathrm{C}, \\
20+10 \min \end{array}$ & $24 ; 1: 3.5$ \\
\hline 12 & $2 \mathrm{~h}$ & $3 d$ & $\mathbf{1 a}(3+1)$ & $4 i$ & $\begin{array}{l}\mathrm{ClCH}_{2} \mathrm{CH}_{2} \mathrm{Cl}, \\
100{ }^{\circ} \mathrm{C}, 20+10 \\
\min \end{array}$ & $24 ; 1: 3.4$ & $\begin{array}{l}\mathrm{ClCH}_{2} \mathrm{CH}_{2} \mathrm{Cl}, \\
100{ }^{\circ} \mathrm{C}, 20+10 \\
\min \end{array}$ & $22 ; 1: 3.9$ \\
\hline 13 & $2 \mathrm{~h}$ & $3 d$ & $\mathbf{1 b}(3+1)$ & $4 i$ & $\begin{array}{l}\mathrm{CH}_{2} \mathrm{Cl}_{2}, 100{ }^{\circ} \mathrm{C}, \\
20+10 \min \end{array}$ & $79 ; 1: 3.1$ & $\begin{array}{l}\mathrm{CH}_{2} \mathrm{Cl}_{2}, 100{ }^{\circ} \mathrm{C}, \\
20+10 \min \end{array}$ & $89 ; 1: 3.3$ \\
\hline 14 & $2 \mathrm{~h}$ & $3 d$ & $\mathbf{1 b}(3+1)$ & $4 i$ & $\begin{array}{l}\mathrm{ClCH}_{2} \mathrm{CH}_{2} \mathrm{Cl}, \\
100{ }^{\circ} \mathrm{C}, 20+10 \\
\min \end{array}$ & $70 ; 1: 3.6$ & $\begin{array}{l}\mathrm{ClCH}_{2} \mathrm{CH}_{2} \mathrm{Cl}, \\
100{ }^{\circ} \mathrm{C}, 20+10 \\
\min \end{array}$ & $90 ; 1: 3.0$ \\
\hline 15 & $2 \mathrm{~h}$ & 3d & $1 \mathrm{~b}(4)$ & $4 \mathbf{i}$ & Not tested & - & $\begin{array}{l}\mathrm{ClCH}_{2} \mathrm{CH}_{2} \mathrm{Cl}, \\
100{ }^{\circ} \mathrm{C}, 30 \mathrm{~min}\end{array}$ & $83 ; 1: 3.8$ \\
\hline
\end{tabular}

${ }^{a}$ Yields of isolated homogeneous product. $E / Z$ ratio was determined by NMR analysis of the crude mixture.

${ }^{\mathrm{b}}$ The times indicated does not include ramp up time (which is $20-80 \mathrm{~s}$ depending on conditions)

${ }^{c}$ nd $=$ not determined

Paralleling the remark of professor Hoveyda, ${ }^{3 \mathrm{f}}$ acrylonitrile might be categorised as a Type III olefin with catalyst $\mathbf{1 b}$ and Type IV with 1a (although it is not truly spectator to CM) in the Grubbs' caterorisation of 
olefins. $^{1 \mathrm{~d}}$ Trace amount $(<0.1 \%)$ of the $(Z)$ homodimer of acrylonitrile (3d) are detected with both catalyst $\mathbf{1} \mathbf{a}, \mathbf{b}$, and a minor amount $(<5 \%)$ of the (E)-homodimer of the substrate are also observed.

Through this series of comparative CM reactions performed under classical thermal conditions and under microwave irradiation, the beneficial effect of microwave activation is clear, particularly in reluctant cases. However, a microwave effect could not be evidenced. ${ }^{4 \mathrm{c}, \mathrm{q}}$ The better yields and conversions observed under microwave irradiation appear to result from the

Dichloromethane and dichloroethane were dried by refluxing with calcium hydride and then distilled under an argon atmosphere. The reactions were monitored by TLC, which were performed on Merck 60F254 plates and visualised with an ethanolic solution of $p$ anisaldehyde and sulfuric acid or an ethanolic solution of molybdophosphoric acid. Flash chromatography was performed with Merck 40-63 $\mu \mathrm{m}$ silica gel eluted with diethyl ether or ethyl acetate in petrol ether. NMR data were recorded on a Bruker Avance 300 spectrometer in $\mathrm{CDCl}_{3}$ and chemical shifts $(\delta)$ are given in ppm relative to the residual $\mathrm{CHCl}_{3}$ signal for ${ }^{1} \mathrm{H} \mathrm{NMR}(7.26 \mathrm{ppm})$ and relative to the deutered solvent signal for ${ }^{13} \mathrm{C}$ NMR (77.0 ppm); coupling constants $(J)$ are in Hertz, and the classical abbreviations are used to describe the signal multiplicity. Mass spectra were recorded on a API III Plus Sciex spectrometer, or an Applied Biosystems 3200 Qtrap both equipped with an ESI source. AllylTMS, allyl acetate, 1-hexene, 4-bromo-1-butene, 5-nitro-1-pentene and catalysts 1a,b were used as received (Aldrich). Acrylonitrile was distilled prior use. $\mathbf{2 a - d}$ and $\mathbf{2 g}$ were obtained by standard allylation methodology from the corresponding activated ketones and allyl bromide (2b,c and 2g: $\mathrm{K}_{2} \mathrm{CO}_{3}$, acetone; 2a: $\mathrm{LiOH}, \mathrm{THF} ; \mathbf{2 d}$ : $\mathrm{NaH}, \mathrm{DMF}$ ), and $\mathbf{2 h}$ was obtained by trans-esterification of the corresponding ethyl ester (Aldrich) with 3-buten-1-ol (DMAP, toluene).

General procedure for thermal solvent-free $\mathrm{CM}$ reactions (4a-c and 4e,g): Neat $2 \mathrm{a}-\mathrm{c}$ or $\mathbf{2 e , g}(1.0 \mathrm{mmol})$, the appropriate alkene $\mathbf{3 a}, \mathbf{b}(3.0 \mathrm{mmol})$ and the required amount of catalyst 1a (see Table 1) were placed in a small sealed tubular reaction vessel $(7 \mathrm{~mL})$ equipped with a teflon coated stirring bar under an argon atmosphere. The reaction vessel was placed in a pre-heated oil bath at the required temperature for the desired time (see Table 1). The excess of alkene $\mathbf{3 a}, \mathbf{b}$ was evaporated under reduced pressure and the residue was purified by flash chromatography to afford pure $\mathbf{4 a - c}$ or $\mathbf{4 e , g}$.

General procedure for thermal CM reactions with solvent (4d and $4 \mathbf{h}, \mathbf{i})$ : A $0.1 \mathrm{M}$ solution of $\mathbf{2 d}$ or $\mathbf{2 g}$, $\mathbf{h}$ $(0.15 \mathrm{mmol})$, the appropriate alkene $\mathbf{3 a}(0.45 \mathrm{mmol})$ or 3c,d $(0.30 \mathrm{mmol}), \mathrm{Ti}(\mathrm{O} i \mathrm{Pr})_{4}(0.023 \mathrm{mmol})$ in the case of entry 5 , and the required amount of catalyst $\mathbf{1 a , b}$ (see Table 1; for entries 11-14, the catalyst was added in two portions: $3 \mathrm{~mol} \%$ at $\mathrm{t}=0$, and $1 \mathrm{~mol} \%$ at $\mathrm{t}=20 \mathrm{~min}$ ) rapid heating allowed in the microwave oven, and a faster cross-metathesis reaction relative to catalyst decomposition. In some cases, the latter effect allowed a substantial decrease of the amount of catalyst.

In conclusion, we have demonstrated that microwave irradiation does not only dramatically reduce the reaction times of cross-metathesis, but also allows to obtain higher TON of the catalysts and ultimately render efficient otherwise unproductive reactions.

were placed in a small sealed tubular reaction vessel (7 $\mathrm{mL}$ ) equipped with a teflon coated stirring bar under an argon atmosphere. The reaction vessel was placed in a pre-heated oil bath at the required temperature for the desired time (see Table 1). The solvent and the excess of alkene 3a or 3c,d were evaporated under reduced pressure and the residue was purified by flash chromatography to afford pure $\mathbf{4 d}$ or $\mathbf{4 h}, \mathbf{i}$.

General procedure for microwave assisted CM reactions (4a-i): Microwave irradiations were performed in a CEM Discover 1-300W oven in sealed tubes $(10 \mathrm{~mL})$ equipped with a teflon coated stirring bar under argon at the temperature and times shown in Table 1 (mode discover standard). The reaction mixtures were prepared as described above, except in the cases of entries 1 and 2 where 1 ,4-benzoquinone $(0.1 \mathrm{mmol}, 10 \mathrm{~mol} \%)$ was added. The products were purified as described above.

4a: colorless oil; $E / Z=3: 1$

${ }^{1} \mathrm{H}$ NMR (mixture of isomers) $\delta:-0.03 /-0.01(\mathrm{~s} / \mathrm{s}, 9 \mathrm{H})$, $1.08(\mathrm{t}, J=7.1 \mathrm{~Hz}, 6 \mathrm{H}), 1.40(\mathrm{~d}, J=8.0 \mathrm{~Hz}, 1 \mathrm{H}), 1.44$ $(\mathrm{d}, J=9.2 \mathrm{~Hz}, 1 \mathrm{H}), 1.56-2.06(\mathrm{~m}, 3 \mathrm{H}), 2.06-2.70(\mathrm{~m}$, $5 \mathrm{H}), 3.14-3.55(\mathrm{~m}, 4 \mathrm{H}), 5.05-5.25(\mathrm{~m}, 1 \mathrm{H}), 5.33-5.56$ $(\mathrm{m}, 1 \mathrm{H})$.

${ }^{13} \mathrm{C}$ NMR ((E) isomer) $\delta:-1.6\left(\mathrm{CH}_{3}\right), \quad 13.2\left(\mathrm{CH}_{3}\right)$, 18.9 $\left(\mathrm{CH}_{2}\right), 31.2\left(\mathrm{CH}_{2}\right), 33.9\left(\mathrm{CH}_{2}\right), 34.0\left(\mathrm{CH}_{2}\right), 37.1\left(\mathrm{CH}_{2}\right)$, 41.0 $\left(\mathrm{CH}_{2}\right), 61.2(\mathrm{C}), 121.5(\mathrm{CH}), 128.4(\mathrm{CH}), 168.5(\mathrm{C})$, 215.5(C).

${ }^{13} \mathrm{C}$ NMR ((Z) isomer) $\delta:-1.8\left(\mathrm{CH}_{3}\right), \quad 13.2\left(\mathrm{CH}_{3}\right)$, 18.5 $\left(\mathrm{CH}_{2}\right), 23.0\left(\mathrm{CH}_{2}\right), 33.6\left(\mathrm{CH}_{2}\right)$, 7.2 $\left(\mathrm{CH}_{2}\right), 37.3\left(\mathrm{CH}_{2}\right)$, 41.0 $\left(\mathrm{CH}_{2}\right), 61.6(\mathrm{C}), 123.1(\mathrm{CH}), 130.7(\mathrm{CH}), 168.6(\mathrm{C})$, 215.8(C).

MS (ESI+): $m / z=310[\mathrm{M}+\mathrm{H}]^{+}, 332[\mathrm{M}+\mathrm{Na}]^{+}, 348$ $[\mathrm{M}+\mathrm{K}]^{+}$.

4b: colorless oil; $E / Z=1: 1$

${ }^{1} \mathrm{H}$ NMR (mixture of isomers) $\delta:-0.07(\mathrm{~s}, 9 \mathrm{H}), 1.20-1.45$ $(\mathrm{m}, 2 \mathrm{H}), 1.50-1.84(\mathrm{~m}, 2 \mathrm{H}), 1.85-2.84(\mathrm{~m}, 7 \mathrm{H}), 3.01$, (ddm, $J=6.2,2.4 \mathrm{~Hz}, 1 \mathrm{H}), 4.76-5.00(\mathrm{~m}, 1 \mathrm{H}), 5.25-5.62$ (m, 1H), 7.42-7.75 (m, 5H).

${ }^{13} \mathrm{C}$ NMR (mixture of isomers) $\delta:-2.0\left(\mathrm{CH}_{3}\right),-1.8\left(\mathrm{CH}_{3}\right)$, 18.7 $\left(\mathrm{CH}_{2}\right), 21.4\left(\mathrm{CH}_{2}\right), 21.5\left(\mathrm{CH}_{2}\right), 23.0\left(\mathrm{CH}_{2}\right), 25.1\left(\mathrm{CH}_{2}\right)$, 25.1 $\left(\mathrm{CH}_{2}\right), 29.4\left(\mathrm{CH}_{2}\right), 29.5\left(\mathrm{CH}_{2}\right), 31.2\left(\mathrm{CH}_{2}\right), 37.1\left(\mathrm{CH}_{2}\right)$, $41.6\left(\mathrm{CH}_{2}\right), \quad 41.6\left(\mathrm{CH}_{2}\right), \quad 75.6(\mathrm{C}), 75.8(\mathrm{C}), 119.6(\mathrm{CH})$, $120.7(\mathrm{CH}), \quad 128.6(\mathrm{CH}), \quad 130.2(\mathrm{CH}), \quad 130.2(\mathrm{CH})$, $130.6(\mathrm{CH}), \quad 130.6(\mathrm{CH}), \quad 133.0(\mathrm{CH}), \quad 134.0(\mathrm{CH})$, 134.0(CH), 135.4(C), 135.4(C), 204.8(C), 204.8(C). 
MS (ESI+): $m / z=365[\mathrm{M}+\mathrm{H}]^{+}, 382\left[\mathrm{M}+\mathrm{NH}_{4}\right]^{+}, 387$ $[\mathrm{M}+\mathrm{Na}]^{+}, 403[\mathrm{M}+\mathrm{K}]^{+}$.

4c: colorless oil; $E / Z=1.8 / 1$

${ }^{1} \mathrm{H}$ NMR (mixture of isomers) $\delta:-0.04 /-0.02(\mathrm{~s} / \mathrm{s}, 9 \mathrm{H})$, 1.17-1.27 (m, 4H), $1.38(\mathrm{~d}, J=8.1 \mathrm{~Hz}, 2 \mathrm{H}), 1.44(\mathrm{~d}, J=$ $8.1 \mathrm{~Hz}, 2 \mathrm{H}), 1.73-1.90(\mathrm{~m}, 1 \mathrm{H}), 2.43-2.51(\mathrm{~m}, 2 \mathrm{H}), 2.52-$ $2.74(\mathrm{~m}, 4 \mathrm{H}), 4.92-5.06(\mathrm{~m}, 1 \mathrm{H}), 5.35-5.57(\mathrm{~m}, 1 \mathrm{H})$.

${ }^{13} \mathrm{C}$ NMR (mixture of isomers) $\delta:-1.9\left(\mathrm{CH}_{3}\right),-1.6\left(\mathrm{CH}_{3}\right)$, 17.8 $\left(\mathrm{CH}_{3}\right), 17.8\left(\mathrm{CH}_{3}\right), 18.5\left(\mathrm{CH}_{2}\right), 18.8\left(\mathrm{CH}_{2}\right), 19.2\left(\mathrm{CH}_{2}\right)$, 23.2 $\left(\mathrm{CH}_{2}\right), 35.4\left(\mathrm{CH}_{2}\right), 38.3\left(\mathrm{CH}_{2}\right), 38.4\left(\mathrm{CH}_{2}\right), 41.6\left(\mathrm{CH}_{2}\right)$, 65.4(C), 66.2(C), 120.4(CH), 121.4(CH), 130.0(CH), 132.0(CH), 210.0(C), 210.5(C).

MS (ESI+): $m / z=253[\mathrm{M}+\mathrm{H}]^{+}, 270\left[\mathrm{M}+\mathrm{NH}_{4}\right]^{+}, 275$ $[\mathrm{M}+\mathrm{Na}]^{+}, 291[\mathrm{M}+\mathrm{K}]^{+}$.

4d: light brown oil; $E / Z=1.8 / 1$

${ }^{1} \mathrm{H}$ NMR (mixture of isomers) $\delta:-0.03 / 0.00(\mathrm{~s} / \mathrm{s}, 9 \mathrm{H})$, $1.45(\mathrm{t}, J=7.0 \mathrm{~Hz}, 2 \mathrm{H}), 1.62-1.92(\mathrm{~m}, 4 \mathrm{H}), 1.92-2.14$ $(\mathrm{m}, 1 \mathrm{H}), 2.46-2.71(\mathrm{~m}, 3 \mathrm{H}), 2.72-2.95(\mathrm{~m}, 2 \mathrm{H}), 5.03-$ $5.24(\mathrm{~m}, 1 \mathrm{H}), 5.41-5.77(\mathrm{~m}, 1 \mathrm{H})$.

${ }^{13} \mathrm{C}$ NMR (mixture of isomers) $\delta:-2.0\left(\mathrm{CH}_{3}\right),-1.8\left(\mathrm{CH}_{3}\right)$, 18.6 $\left(\mathrm{CH}_{2}\right), 21.4\left(\mathrm{CH}_{2}\right), 21.4\left(\mathrm{CH}_{2}\right), 23.2\left(\mathrm{CH}_{2}\right), 26.8\left(\mathrm{CH}_{2}\right)$, 26.9 $\left(\mathrm{CH}_{2}\right), 32.9\left(\mathrm{CH}_{2}\right), 35.8\left(\mathrm{CH}_{2}\right), 36.0\left(\mathrm{CH}_{2}\right), 39.1\left(\mathrm{CH}_{2}\right)$, 39.7 $\left(\mathrm{CH}_{2}\right), \quad 39.8\left(\mathrm{CH}_{2}\right), 96.7(\mathrm{C}), 97.3(\mathrm{C}), 118.1(\mathrm{CH})$, 119.3(CH), 131.6(CH), 133.7(CH), 200.5(C), 200.6(C).

MS: $m / z=270[\mathrm{M}+\mathrm{H}]^{+}, 287\left[\mathrm{M}+\mathrm{NH}_{4}\right]^{+}, 292[\mathrm{M}+\mathrm{Na}]^{+}$, $308[\mathrm{M}+\mathrm{K}]^{+}$.

4e: colorless oil; $E / Z=1.9 / 1$

${ }^{1} \mathrm{H}$ NMR (mixture of isomers) $\delta: 0.00 / 0.01(\mathrm{~s} / \mathrm{s}, 9 \mathrm{H})$, $1.44(\mathrm{~d}, J=8.2 \mathrm{~Hz}, 1.3 \mathrm{H}), 1.49(\mathrm{q}, J=7.7 \mathrm{~Hz}, 0.7 \mathrm{H})$, 2.49-2.62 (m, 2H), $3.35(\mathrm{t}, J=7.2 \mathrm{~Hz}, 2 \mathrm{H}), 5.16-5.32$ $(\mathrm{m}, 1 \mathrm{H}), 5.46-5.62(\mathrm{~m}, 1 \mathrm{H})$.

${ }^{13} \mathrm{C}$ NMR ((E) isomer) $\delta:-1.9\left(\mathrm{CH}_{3}\right), \quad 23.0\left(\mathrm{CH}_{2}\right)$, 33.6 $\left(\mathrm{CH}_{2}\right), 36.4\left(\mathrm{CH}_{2}\right), 124.9(\mathrm{CH}), 130.3(\mathrm{CH})$.

${ }^{13} \mathrm{C} \quad \mathrm{NMR} \quad((\mathrm{Z})$ isomer $) \delta: \quad-1.7\left(\mathrm{CH}_{3}\right), \quad 19.0\left(\mathrm{CH}_{2}\right)$, 30.9 $\left(\mathrm{CH}_{2}\right), 32.7\left(\mathrm{CH}_{2}\right), 123.6(\mathrm{CH}), 129.0(\mathrm{CH})$.

MS: $m / z=221 / 223[\mathrm{M}+\mathrm{H}]^{+}, 238 / 240\left[\mathrm{M}+\mathrm{NH}_{4}\right]^{+}$, 243/245 [M+Na $]^{+}, 259 / 261[\mathrm{M}+\mathrm{K}]^{+}$.

4f: colorless oil; $E / Z=2.3 / 1$

${ }^{1} \mathrm{H}$ NMR (mixture of isomers) $\delta:-0.02 /-0.01(\mathrm{~s} / \mathrm{s}, 9 \mathrm{H})$, 1.40-1.47 (m, 2H), 2.00-2.12 (m, 4H), 4.32-4.42 (m, $2 \mathrm{H}), 5.05-5.25(\mathrm{~m}, 1 \mathrm{H}), 5.36-5.54(\mathrm{~m}, 1 \mathrm{H})$.

${ }^{13} \mathrm{C}$ NMR ((E) isomers) $\delta:-1.9\left(\mathrm{CH}_{3}\right), \quad 22.9\left(\mathrm{CH}_{2}\right)$, $27.6\left(\mathrm{CH}_{2}\right), \quad 29.4\left(\mathrm{CH}_{2}\right), \quad 74.9\left(\mathrm{CH}_{2}\right), \quad 125.5(\mathrm{CH})$, 129.3(CH).

${ }^{13} \mathrm{C}$ NMR ((Z) isomers) $\delta: \quad-1.7\left(\mathrm{CH}_{3}\right), \quad 18.7\left(\mathrm{CH}_{2}\right)$, $23.7\left(\mathrm{CH}_{2}\right), \quad 27.4\left(\mathrm{CH}_{2}\right), \quad 75.1\left(\mathrm{CH}_{2}\right), \quad 124.2(\mathrm{CH})$, 128.3(CH).

4g: colorless oil; $E / Z=4 / 1$

${ }^{1} \mathrm{H}$ NMR $((E)$ isomer) $\delta: 0.80-0.90(\mathrm{~m}, 2 \mathrm{H}), 1.22(\mathrm{t}, J=$ $7.2 \mathrm{~Hz}, 3 \mathrm{H}), 1.25-1.35(\mathrm{~m}, 3 \mathrm{H}), 1.78-2.09(\mathrm{~m}, 6 \mathrm{H}), 2.10-$ $2.48(\mathrm{~m}, 5 \mathrm{H}), 2.56(\mathrm{ddd}, J=1.0,7.2,13.8 \mathrm{~Hz}, 1 \mathrm{H}), 4.12$ $(\mathrm{q}, J=7.2 \mathrm{~Hz}, 2 \mathrm{H}), 5.24(\mathrm{ttd}, J=1.0,7.2,15.4 \mathrm{~Hz}, 1 \mathrm{H})$, $5.47(\mathrm{ttd}, J=1.0,6.7,15.4 \mathrm{~Hz}, 1 \mathrm{H})$.
${ }^{13} \mathrm{C}$ NMR $((E)$ isomer $) \quad \delta: \quad 13.9\left(\mathrm{CH}_{3}\right), \quad 14.1\left(\mathrm{CH}_{3}\right)$, 19.5 $\left(\mathrm{CH}_{2}\right), 22.2\left(\mathrm{CH}_{2}\right), 31.5\left(\mathrm{CH}_{2}\right), 32.0\left(\mathrm{CH}_{2}\right), 32.3\left(\mathrm{CH}_{2}\right)$, 36.7 $\left(\mathrm{CH}_{2}\right), 38.2\left(\mathrm{CH}_{2}\right), 60.3(\mathrm{C}), 61.4\left(\mathrm{CH}_{2}\right), 124.1(\mathrm{CH})$, 135.1(CH), 171.1(C), 214.8(C).

MS: $m / z=239[\mathrm{M}+\mathrm{H}]^{+}, 261[\mathrm{M}+\mathrm{Na}]^{+}, 277[\mathrm{M}+\mathrm{K}]^{+}$.

4h: colorless oil; $E / Z=1 / 5.7$

${ }^{1} \mathrm{H}$ NMR $((Z)$ isomer) $\delta: 1.22(\mathrm{t}, J=7.2 \mathrm{~Hz}, 3 \mathrm{H}), 1.82-$ $1.98(\mathrm{~m}, 3 \mathrm{H}), 2.02(\mathrm{~s}, 3 \mathrm{H}), 2.20-2.32(\mathrm{~m}, 1 \mathrm{H}), 2.32-2.48$ $(\mathrm{m}, 3 \mathrm{H}), 2.59-2.69(\mathrm{~m}, 1 \mathrm{H}), 4.13(\mathrm{q}, J=7.2 \mathrm{~Hz}, 2 \mathrm{H})$, $4.47(\mathrm{~d}, J=4.6 \mathrm{~Hz}, 2 \mathrm{H}), 5.63(\mathrm{~m}, 2 \mathrm{H})$.

${ }^{13} \mathrm{C}$ NMR $((\mathrm{Z})$ isomer $) \delta: 14.2\left(\mathrm{CH}_{3}\right), \quad 19.6\left(\mathrm{CH}_{2}\right)$, 21.0 $\left(\mathrm{CH}_{3}\right), 32.3\left(\mathrm{CH}_{2}\right), 36.3\left(\mathrm{CH}_{2}\right), 38.1\left(\mathrm{CH}_{2}\right), 59.9(\mathrm{C})$, 61.6 $\left(\mathrm{CH}_{2}\right), 64.7\left(\mathrm{CH}_{2}\right), 128.6(\mathrm{CH}), 130.0(\mathrm{CH}), 170.8(\mathrm{C})$, 170.9(C), 214.5(C).

MS: $m / z=286\left[\mathrm{M}+\mathrm{NH}_{4}\right]^{+}, 291[\mathrm{M}+\mathrm{Na}]^{+}, 307[\mathrm{M}+\mathrm{K}]^{+}$.

4i: colorless oil; $E / Z=1: 2.4$

${ }^{1} \mathrm{H}$ NMR (mixture of isomers) $\delta$ : 1.78-1.91 (m, $\left.1 \mathrm{H}\right)$, 2.04-2.18 (m, 1H), 2.20-2.33 (m, 4H), 2.55 (pseudo q, $J$ $=6.4 \mathrm{~Hz}, 1.4 \mathrm{H}$ ), 2.74 (pseudo q, $J=6.4 \mathrm{~Hz}, 0.6 \mathrm{H}$ ), 3.12 $(\mathrm{dd}, J=9.2,9.0 \mathrm{~Hz}, 0.7 \mathrm{H}), 3.13(\mathrm{dd}, J=9.5,9.0 \mathrm{~Hz}$, $0.3 \mathrm{H}), 4.08-4.30(\mathrm{~m}, 2 \mathrm{H}), 5.43(\mathrm{dd}, J=10.8,1.3 \mathrm{~Hz}$, $0.7 \mathrm{H}), 5.44(\mathrm{dd}, J=16.4,1.5 \mathrm{~Hz}, 0.3 \mathrm{H}), 6.68(\mathrm{ddd}, J=$ $16.4,7.1,6.9 \mathrm{~Hz}, 0.7 \mathrm{H}), 6.52(\mathrm{ddd}, J=10.8,7.7,7.4 \mathrm{~Hz}$, $0.3 \mathrm{H})$.

${ }^{13} \mathrm{C}$ NMR ((Z) isomer) $\delta: \quad 20.8\left(\mathrm{CH}_{2}\right), \quad 27.1\left(\mathrm{CH}_{2}\right)$, 31.1 $\left(\mathrm{CH}_{2}\right), 37.9\left(\mathrm{CH}_{2}\right), 54.6(\mathrm{CH}), 62.4\left(\mathrm{CH}_{2}\right), 102.0(\mathrm{CH})$, 115.4(C), 149.9(CH), 169.0(C), 212.0(C).

${ }^{13} \mathrm{C}$ NMR ((E) isomer) $\delta: \quad 20.7\left(\mathrm{CH}_{2}\right), \quad 27.0\left(\mathrm{CH}_{2}\right)$, 32.4 $\left(\mathrm{CH}_{2}\right), 37.8\left(\mathrm{CH}_{2}\right), 54.5(\mathrm{CH}), 62.2\left(\mathrm{CH}_{2}\right), 102.3(\mathrm{CH})$, 116.8(C), 150.7(CH), 169.0(C), 211.8(C).

$\mathrm{MS}(\mathrm{ESI}+): m / z=208[\mathrm{M}+\mathrm{H}]^{+}, 230[\mathrm{M}+\mathrm{Na}]^{+}, 246$ $[\mathrm{M}+\mathrm{K}]^{+}$.

\section{Acknowledgment}

We thank the French Research Ministry for fellowship awards to T.B. and A.M., the Université Paul Cézanne, the CNRS (UMR 6178) and the Conseil Général des Bouches du Rhônes for financial support.

\section{References}

(1) For reviews on CM, see: (a) Vernall, A. J.; Abell, A. D. Aldrichimica Acta 2003, 36, 93-105. (b) Connon, S. J.; Blechert, S. Angew. Chem., Int. Ed. 2003, 42, 1900-1923. (c) Blackwell, H. E.; O’Leary, D. J.; Chatterjee, A. K.; Washenfelder, R. A.; Bussmann, D. A.; Grubbs, R. H. J. Am. Chem. Soc. 2000, 122, 58-71. For a general model for selectivity in olefin CM, see: (d) Chatterjee, A. K.; Choi, T.-L.; Sanders, D. P.; Grubbs, R. H. J. Am. Chem. Soc. 2003, 125, 11360-11370.

(2) Vedrenne, E.; Dupont, H.; Oualef, S.; Elkaïm, L.; Grimaud, L. Synlett 2005, 670-672.

(3) (a) Randl, S.; Gessler, S.; Wakamatsu, H.; Blechert, S. Synlett 2001, 430-432. (b) Love, J. A.; Morgan, J. P.; Trnka, T. M.; Grubbs, R. H. Angew. Chem., Int. Ed. 2002, 41, 4035-4037. (c) Rivard, M.; Blechert, S. Eur. J. Org. Chem. 2003, 2225-2228. (d) Michrowska, A.; Bujok, R.; Harutyunyan, S.; Sashuk, V.; Dolgonos, G.; Grela, K. J. 
Am. Chem. Soc. 2004, 126, 9318-9325. (e) Bujok, R.; Bieniek, M.; Masnyk, M.; Michrowska, A.; Sarosiek, A.; Stepowska, H.; Arlt, D.; Grela, K. J. Org. Chem. 2004, 69, 6894-6896. (f) Hoveyda, H. R.; Vézina, M. Org. Lett. 2005, 7, 2113-2116. (g) Bai, C.-X.; Zhang, W.-Z.; He, R.; Lu, X.-B.; Zhang, Z.-Q. Tetrahedron Lett. 2005, 46, 72257228. (h) Bai, C.-X.; Lu, X.-B.; He, R.; Zhang, W.-Z.; Feng, X.-J. Org. Biomol. Chem. 2005, 3, 4139-4142. For $\mathrm{CM}$ of acrylonitrile with molybdenum based catalyst, see: (i) Crowe, W. E.; Goldberg, D. R. J. Am. Chem. Soc. 1995, 117, 5162-5163.

(4) For reviews on microwave-assisted synthesis, see: (a) Kappe, C. O. Angew. Chem., Int. Ed. 2004, 43, 6250-6284. (b) Hayes, B. L. Aldrichimica Acta 2004, 37, 66-77. (c) de la Hoz, A.; Díaz-Ortiz, Á.; Moreno, A. Chem. Soc. Rev. 2005, 34, 164-178. For microwave-assisted RCM, see: (d) Varray, S.; Gauzy, C.; Lamaty, F.; Lazaro, R.; Martinez, J. J. Org. Chem. 2000, 65, 6787-6790. (e) Mayo, K. G.; Nearhoof, E. H.; Kiddle, J. J. Org. Lett. 2002, 4, $1567-$ 1570. (f) Garbacia, S.; Desai, B.; Lavastre, O.; Kappe, C. O. J. Org. Chem. 2003, 68, 9136-9139. (g) Thanh, G. V.; Loupy, A. Tetrahedron Lett. 2003, 44, 9091-9094. (h) Grigg, R.; Martin, W.; Morris, J.; Sridharan, V. Tetrahedron Lett. 2003, 44, 4899-4901. (i) Balan, D.; Adolfsson, H. Tetrahedron Lett. 2004, 45, 3089-3092. (j) Comer, E.; Organ, M. G. J. Am. Chem. Soc. 2005, 127, 8160-8167. (k) Nosse, B.; Schall, A.; Jeong, W. B.; Reiser, O. Adv. Synth. Catal. 2005, 347, 1869-1874. (1) Appukkuttan, P.; Dehaen, W.; Van der Eycken, E. Org. Lett. 2005, 7, 2723-2726. (m) Collins, S. K.; Grandbois, A.; Vachon, M. P.; Côté, J. Angew. Chem., Int. Ed. 2006, 45, 2923-2926. (n) Yang, Q. Y.; Li, X.-Y.; Wu, H.; Xiao, W.-J. Tetrahedron Lett. 2006, 47, 3893-3896. (o) Chapman, R. N.; Arora, P. S. Org. Lett. 2006, 8, 5825-5828. For microwave-assisted CM, see: (p) Poulsen, S.-A.; Bornaghi, L. F. Tetrahedron Lett. 2005, 46, 7389-7392. (q) Bargiggia, F. C.; Murray, W. V. J. Org. Chem. 2005, 70, 9636-9639. (r) Morris, T.; Sandham, D.; Caddick, S. Org. Biomol. Chem. 2007, 5, 1025-1027.

(5) Hong, S. H.; Sanders, D. P.; Lee, C. W.; Grubbs, R. H. J. Am. Chem. Soc. 2005, 127, 17160-17161.

(6) As the CM is a reversible process, the TON (turnover number) represents the average number of substrate molecules converted into the cross-product per molecule of catalyst. A typical loading of $\mathbf{1} \mathbf{a}, \mathbf{b}$ in CM reaction is $2-10 \%$, which correspond to a maximum TON of 10-50. For a recent TON study in ruthenium carbene catalyzed ringclosing metathesis, see: Maechling, S.; Zaja, M.; Blechert, S. Adv. Synth. Catal. 2005, 347, 1413-1422. 